\title{
Surface Treatment Agent for Dental Metals using a Thiirane Monomer and a Phosphoric Acid Monomer
}

\author{
Yoshinori KADOMA \\ Department of Applied Functional Molecules, \\ Division of Biofunctional Molecules, \\ Institute of Biomaterials and Bioengineering, \\ Tokyo Medical and Dental University, \\ 2-3-10, Kanda-surugadai, Chiyoda-ku, \\ Tokyo 101-0062, Japan
}

Received January 15, 2002/Accepted March 26, 2002

To develop a new surface treatment agent which improves the bond strength of adhesive resin to both non-precious and precious metals, experimental treatment agents containing both an adhesive bonding promoter for precious metals and one for non-precious metals were prepared by dissolving epithioalkyl methacrylate (EP3MA or EP8MA) and 10-methacryloyloxydecyl dihydrogen phosphate (MDP) in acetone. The surfaces of dental metals were treated by the treatment agents and metal specimens were butt-jointed together with MMA-PMMA resins. After 2,000 thermal cyclings in water at temperatures of 4 and $60^{\circ} \mathrm{C}$, tensile bond strengths were measured. The effectiveness of surface treatments was evaluated by tensile bond strengths and microscopic failure mode analysis after the tensile test.

The combined treatment of EP3MA-MDP or EP8MA-MDP was used effectively for nonprecious metals as well as precious metals, and was shown to be extremely effective compared with the single treatment of EP3MA, EP8MA, or MDP.

Key words: Surface treatment, Thiirane, Phosphoric methacrylate

\section{INTRODUCTION}

Since bonding of resin to dental precious metal alloys is difficult because of their chemical inertness, a number of surface modification methods have been developed, such as high-temperature oxidation ${ }^{1)}$, electroplating with $\operatorname{tin}^{2)}$, silica coating ${ }^{3)}$, and the application of liquid Ga-Sn alloy ${ }^{4}$. Surface treatment with precious metal primer has recently attracted interest as an alternative method to improve the bonding between precious metals and resins, because the application method is simpler and less expensive than other methods, and does not require any proprietary equipment. Laboratory evaluations have demonstrated that some sulfur-containing compounds possess chemical affinity toward gold substrate ${ }^{5)}$ leading to adhesion effects as a primer, and that the derivative monomers significantly improve the bond strength of resin to dental precious metal alloys. Adhesive bonding promoters that have been utilized for precious metal primers include thiol $^{6)}$, thiophenol ${ }^{7)}$, disulfide ${ }^{8)}$, thiophosphoric acid ${ }^{9}$, thiobarbituric acid ${ }^{10)}$, triazinedithione $\mathrm{e}^{11)}$, sulfide $^{12)}$, dithiolane $\mathrm{e}^{13)}$ and thiouracil ${ }^{14)}$. Thiol and thiophenol lack storage stability because of their high reactivity to double bond, although they are extremely reactive to precious metals, such as gold or palladium. 
It is generally difficult to reconcile the storage stability and the reactivity to precious metals of sulfur-containing compounds. Further, acidic adhesive bonding promoters may undergo acid-base reactions with amine used as a component of the polymerization initiator system, and, therefore, their application methods are limited.

Thiirane ${ }^{15)}$ is a sulfide with a three-membered ring and exhibits satisfactory solubility and storage stability. It is known that thiirane undergoes a chemical reaction on the gold surface involving the cleavage of the episulfide ring and C-S bond formation leading to the production of a thiol-like molecule bound to gold ${ }^{16)}$. Preliminary experiments using MMA-PMMA resin demonstrated the excellent adhesion performance in relation to precious metal alloys, when thiirane monomers were utilized as an active ingredient added to base monomer mixtures as well as to primers ${ }^{17)}$. Thus, thiirane monomer is one of the most promising adhesive bonding promoters for precious metals at present. However, thiirane monomers are ineffective for improving adhesion to non-precious metals, such as titanium, cobalt-chromium alloy, nickelchromium alloy, and stainless steel. From the clinical point of view, it will be very convenient to prepare metal primers for both non-precious and precious metal alloys, because a single-liquid metal primer can be applied uniformly to all kinds of metal adherends. This study therefore evaluated the surface treatment agents containing both an adhesive bonding promoter for precious metals, epithioalkyl methacrylate (EP3MA or EP8MA), and one for non-precious metals, 10-methacryloyloxydecyl dihydrogen phosphate (MDP) ${ }^{18)}$. Surface treatment agents were prepared by dissolving a thiirane monomer and MDP in acetone. Two metal specimens treated by the agent were butt-jointed with MMA-PMMA resin and then tensile bond strengths were measured after 2,000 thermal cyclings in water. The effects of the surface treatment agents on the water durability of the resin bond were discussed.

\section{MATERIALS AND METHODS}

\section{Materials}

Adhesion promoting monomers, 4,5-epithiopentyl methacrylate (EP3MA) ${ }^{15)}$ and 9,10epithiodecyl methacrylate (EP8MA ${ }^{19)}$, were prepared according to previously described methods, and $\mathrm{MDP}^{18)}$ (Kuraray Medical Inc., Tokyo, Japan) was used as received. The structures of these monomers are shown in Fig. 1. Methyl methacrylate (MMA) was purified by distillation under reduced pressure and benzoyl peroxide (BPO) was recrystallized from a chloroform/methanol solvent system. Acetone (dehydrated, Kanto Chemical Co. Inc., Tokyo, Japan), employed as a solvent, and $N, N$-bis(2-hydroxyethyl)-p-toluidine(DEPT)(Tokyo Kasei Kogyo Co., Ltd., Tokyo, Japan) were used without further purification. PMMA powder and an initiator, tri-n-butylborane oxide (TBBO), were obtained from Sun Medical Co., Ltd. (Kyoto, Japan) as a dental adhesive resin, Super-Bond C \& B. Both the manufacturer and composition of the metal specimens used as adherends are given in Table 1. 


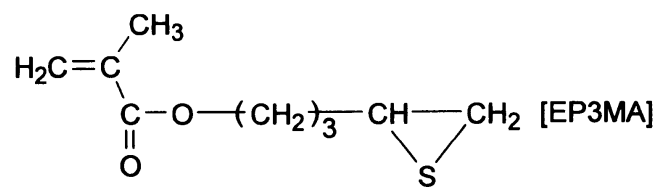

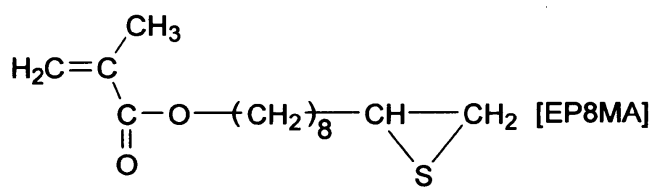

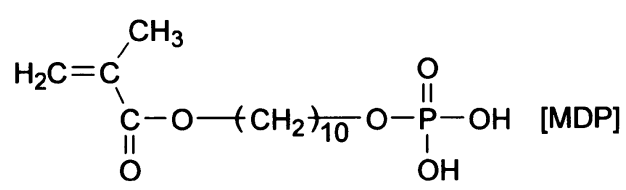

Fig. 1 Structures of EP3MA, EP8MA, and MDP.

Table 1 Composition of dental metals

\begin{tabular}{|c|c|c|c|}
\hline Alloy & Code & Manufacturer & Composition (\%) \\
\hline $\begin{array}{l}\text { Casting Gold } \\
\text { M.C. Type IV }\end{array}$ & $\mathrm{Au}$ alloy & GC & $\mathrm{Au} 70 \quad \mathrm{Cu} 14 \quad \mathrm{Ag} 10 \quad \mathrm{Pt} 3 \quad \mathrm{Pd} 3$ \\
\hline Castwell M.C. & Pd alloy & GC & Ag $45 \quad \mathrm{Pd} 20 \quad \mathrm{Cu} 18 \quad \mathrm{Au} 12$ Others 5 \\
\hline Sunsilver CB & Ag alloy & Sankin & Ag 79 Zn 7 In $7 \quad$ Cu 5 Others 2 \\
\hline Stainless steel & SUS304 & & $\begin{array}{lll}\mathrm{Fe}>70 & \mathrm{Cr} 18 \sim 20 & \mathrm{Ni} \quad 8 \sim 10.5 \\
\mathrm{Mn} \leqq 2 & \text { Others }\end{array}$ \\
\hline Durallium JD & Co-Cr alloy & Jelenko & Co 68 Cr 26 Mo 4 Others 2 \\
\hline $\begin{array}{l}\text { SB bondloy I } \\
\text { Titanium }\end{array}$ & $\begin{array}{l}\mathrm{Ni}-\mathrm{Cr} \text { alloy } \\
\mathrm{Ti}\end{array}$ & Towa Giken & $\begin{array}{l}\text { Ni } 56.4 \text { Cr } 17.4 \quad \text { Mo } 12.4 \text { Fe } 9.9 \text { Others } 3.9 \\
\text { Ti }>99.9\end{array}$ \\
\hline
\end{tabular}

GC (GC Dental Products Corp., Tokyo, Japan), Sankin (Sankin Kogyo Co. Ltd., Tokyo, Japan), Jelenko (Jelenko Dental Health Products, Amonk, USA), Towa Giken (Tokuyama Dental Corp., Tokyo, Japan)

Alloy specimens were cylindrically shaped $(5 \mathrm{~mm} \phi \times 4 \mathrm{~mm})$ and provided an exterior screw at one side which was connected with jigs for tensile test.

\section{Surface treatment agents and experimental resins}

Five surface treatment agents were prepared by dissolving monomer in acetone. Their composition in acetone was $0.1 \mathrm{~mol} \%$ of MDP, $0.1 \mathrm{~mol} \%$ of EP3MA, $0.1 \mathrm{~mol} \%$ of EP8MA, $0.1 \mathrm{~mol} \%$ of EP3MA and $0.1 \mathrm{~mol} \%$ of MDP, or $0.1 \mathrm{~mol} \%$ of EP8MA and $0.1 \mathrm{~mol} \%$ of MDP. Two kinds of initiators, TBBO and BPO-DEPT redox system, were employed for MMA-PMMA experimental resins. In the case of MMA-PMMA/ TBBO resin, TBBO initiator was added to MMA liquid in one dappen dish immediately before use. The polymer powder was placed in another and the resin was applied by the brush-on technique ${ }^{20)}$. MMA-PMMA/BPO-DEPT resin consisted of MMA containing $0.5 \mathrm{wt} \%$ DEPT and PMMA mixed with $1.0 \mathrm{wt} \% \mathrm{BPO}$, and was applied using the same technique. 


\section{Measurement of tensile bond strength to metal adherends}

The surface of cylindrically shaped adherends $(5 \mathrm{~mm} \phi \times 4 \mathrm{~mm})$ was polished to a mirror-like finish with lapping film (Sumitomo 3M Ltd., Tokyo, Japan) using a sequence of \#1,000, \#2,000, and \#4,000 grit. The surface was washed with acetone and dried naturally in air. Five microliters of the surface treatment agent was placed on the adherend surface and allowed to stand for one day. The surface was washed with acetone to remove excessive monomer and allowed to dry prior to bonding. Two specimens of each metal were butt-jointed together with an experimental resin using the brush-on technique. Excess resin outside the circumference of the cylinder was removed using a double-edged razor blade (FEATHER Safety Razor Co. Ltd., Osaka, Japan) and the assembly was left to stand undisturbed for $1 \mathrm{hr}$ at room temperature to allow the adequate cure of the resin. One hour after cementation the bonded specimens were stored in water at room temperature until all of the specimens were ready to be thermocycled. The specimens were then thermocycled 2,000 times in water at temperatures of 4 and $60^{\circ} \mathrm{C}$, with a one-minute dwell time at each temperature. Tensile bond strength measurements were made on 5 specimens for each metal with a universal testing machine (Autograph AGS-1000A, Shimadzu Co., Kyoto, Japan) at a crosshead speed of $2 \mathrm{~mm} / \mathrm{min}$. The mean and standard deviation of 5 replications were calculated for each condition and the results were analyzed at the $95 \%$ level of confidence by the t-test and the three-way analysis of variance with tensile bond strength as a dependent valuable, and with initiator, treatment agent, and adherend as independent factors.

\section{Determination of the area of cohesive failure after the tensile test}

Microscopic photographs of debonded metal surfaces after the tensile test for specimens treated with both a thiirane monomer and MDP in combination were taken at $\times 20$ magnification. Borderlines between cohesive and adhesive failures were drawn on section papers placed on the photographs. Specimens were excluded from the measurement of the area of cohesive failure when the border between cohesive and adhesive failures was unclear. The area of cohesive failure was obtained by counting the number of one-millimeter squares surrounded by borderlines.

\section{RESULTS}

\section{Surface treatment with MDP}

Table 2 lists the bond strengths of MMA-PMMA resins to dental metals treated with MDP alone for two kinds of initiators together with the values without any treatment agent. One day after the application of $0.1 \mathrm{~mol} \%$ of MDP solution to metal surfaces, the surfaces became slightly wet due to evaporation of acetone, and crystallike substances were deposited partly on the surfaces. After rinsing with acetone, the surfaces became indistinguishable from those before MDP application, and, therefore, MDP was considered to be retained in extremely small quantities. As seen in Table 2, many specimens without any treatment agent showed spontaneous adhesive 
failures while thermal cycling. On the other hand, no spontaneous fracture occurred for specimens that used MDP, and the surface treatment with MDP was confirmed to be effective to some extent for precious metal alloys.

\section{Surface treatment with EP3MA or EP8MA}

Tensile bond strengths of MMA-PMMA resins to dental metals treated with either EP3MA or EP8MA in acetone obtained for the two different initiators are summarized in Table 3 . The tensile bond strength to precious metal alloys was significantly enhanced by the thiirane treatment without any spontaneous failures while thermal

Table 2 Tensile bond strength ( $\mathrm{MPa}$ ) of MMA-PMMA resin to dental metals treated with $0.1 \mathrm{~mol} \% \mathrm{MDP}$ in acetone

\begin{tabular}{|c|c|c|c|c|}
\hline \multirow{2}{*}{ Adherend } & \multicolumn{2}{|c|}{ TBBO } & \multicolumn{2}{|c|}{ BPO-DEPT } \\
\hline & Without & MDP & Without & MDP \\
\hline $\mathrm{Au}$ alloy & 2.0 (1.1) [0] & $16.4(6.9)[0]$ & $2.6(0.2)[3]$ & $9.4(5.8)[0]$ \\
\hline Pd alloy & $2.0(0.8)[0]$ & $39.6(1.8)[0]$ & $0.5(0.1)[2]$ & $18.1(1.5)[0]$ \\
\hline $\mathrm{Ag}$ alloy & $0.0 \quad[4]$ & $39.1(4.2)[0]$ & $0.1(0.1)[3]$ & $23.7(5.3)[0]$ \\
\hline SUS304 & [5] & $45.7(4.2)[0]$ & [5] & $42.7(2.2)[0]$ \\
\hline $\mathrm{Co}-\mathrm{Cr}$ alloy & [5] & $32.9(3.0)[0]$ & [5] & $34.1(3.7)[0]$ \\
\hline $\mathrm{Ni}-\mathrm{Cr}$ alloy & [5] & $41.1(1.1)[0]$ & [5] & $38.8(6.0)[0]$ \\
\hline $\mathrm{Ti}$ & [5] & $37.9(1.8)[0]$ & [5] & $38.7(4.7)[0]$ \\
\hline
\end{tabular}

The values in brackets are the number of specimens fractured while thermal cycling, and the specimens are excluded from the calculation of the average and standard deviation.

Treatment agent remaining on the metal surface was washed off before the application of resin.

Tensile bond strength after 2,000 thermal cyclings in water at 4 and $60^{\circ} \mathrm{C}$.

( ): SD

Table 3 Tensile bond strength $(\mathrm{MPa})$ of MMA-PMMA resin to dental metals treated with $0.1 \mathrm{~mol} \%$ EP3MA or $0.1 \mathrm{~mol} \%$ EP8MA in acetone

\begin{tabular}{|c|c|c|c|c|}
\hline \multirow{2}{*}{ Adherend } & \multicolumn{2}{|c|}{ TBBO } & \multicolumn{2}{|c|}{ BPO-DEPT } \\
\hline & EP3MA & EP8MA & EP3MA & EP8MA \\
\hline $\mathrm{Au}$ alloy & $43.1(3.4)[0]$ & $36.1(3.3)[0]$ & $43.5(4.8)[0]$ & $40.1(4.9)[0]$ \\
\hline Pd alloy & $40.9(2.5)[0]$ & $35.4(3.7)[0]$ & $42.5(3.1)[0]$ & $40.0(4.3)[0]$ \\
\hline $\mathrm{Ag}$ alloy & $31.2(2.0)[0]$ & $30.4(4.2)[0]$ & $26.3(4.7)[0]$ & $32.8(5.1)[0]$ \\
\hline SUS304 & $-\quad[5]$ & $-\quad[5]$ & $-\quad[5]$ & $-\quad[5]$ \\
\hline Co-Cr alloy & [5] & [5] & [5] & [5] \\
\hline $\mathrm{Ni}-\mathrm{Cr}$ alloy & [5] & [5] & [5] & [5] \\
\hline $\mathrm{Ti}$ & $1.3(0.8)[2]$ & $1.1(0.5)[0]$ & $4.6(2.4)[3]$ & $5.6(2.6)[1]$ \\
\hline
\end{tabular}

The values in brackets are the number of specimens fractured while thermal cycling, and the specimens are excluded from the calculation of the average and standard deviation.

Treatment agent remaining on the metal surface was washed off before the application of resin.

Tensile bond strength after 2,000 thermal cyclings in water at 4 and $60^{\circ} \mathrm{C}$.

( ): SD 
cycling compared with controls as was the case of ethanol solution ${ }^{15,19)}$. On the other hand, no effect was demonstrated when non-precious metal alloys or Ti were employed as an adherend. The three-way analysis of variance for the single treatment revealed that one factor, adherend $(p<0.001)$, and 2 interactions, initiator $\times$ treatme nt agent $(\mathrm{p}<0.05)$ and treatment agent $\times$ adherend $(\mathrm{p}<0.001)$ were significant. Although the values of the mean bond strength to precious metal alloys were higher in BPO-DEPT initiator than in TBBO initiator with only one exception, the Ag alloy treated by EP3MA, no significant effect existed for the initiator or treatment agent.

\section{Surface treatment using EP3MA or EP8MA with MDP in combination}

The results of the tensile tests for MMA-PMMA/TBBO or MMA-PMMA/BPO-DEPT resin bonded to metal adherends treated by surface treatment agent containing both $0.1 \mathrm{~mol} \%$ of thiirane monomer and $0.1 \mathrm{~mol} \%$ of MDP in acetone for two different initiator systems are summarized in Table 4. The relative proportion of the area of cohesive failure to the total cross-sectional area of the fractured specimens was measured after the tensile test for the combined treatment of EP3MA-MDP or EP8MA-MDP. The results are exhibited graphically in Fig. 2. The three-way analysis of variance for the combined treatment revealed that one factor, adherend $(\mathrm{p}<0.001)$, and 2 interactions, initiator $\times$ treatment agent $(\mathrm{p}<0.001)$ and treatment agent $\times$ adherend $(p<0.01)$ were significant. In the case of the treatment using EP3MA with MDP in combination, tensile bond strengths were highly improved by the use of the treatment agent for all metal adherends, compared with the case without any treatment agent. The values of mean bond strength obtained using TBBOinitiated resin were higher than those of BPO-DEPT-initiated resin, and significant differences were observed between the two resins for $\mathrm{Au}$ alloy, $\mathrm{Co}-\mathrm{Cr}$ alloy and $\mathrm{Ni}-\mathrm{Cr}$

Table 4 Tensile bond strength ( $\mathrm{MPa}$ ) of MMA-PMMA resin to dental metals treated with the combined use of 0.1 mol\% EP3MA and $0.1 \mathrm{~mol} \%$ MDP in acetone or $0.1 \mathrm{~mol} \%$ EP8MA and $0.1 \mathrm{~mol} \% \mathrm{MDP}$ in acetone

\begin{tabular}{lcccccc}
\hline \multirow{2}{*}{ Adherend } & \multicolumn{3}{c}{ TBBO } & & \multicolumn{2}{c}{ BPO-DEPT } \\
\cline { 2 - 3 } \cline { 5 - 7 } & EP3MA+MDP & EP8MA+MDP & & EP3MA+MDP & EP8MA+MDP \\
\hline Au alloy & $43.0(5.4)[0]$ & $43.5(2.9)[0]$ & & $34.9(3.0)[0]$ & $42.4(5.1)[0]$ \\
Pd alloy & $43.3(2.1)[0]$ & $44.5(1.1)[0]$ & & $42.5(5.4)[0]$ & $46.7(1.6)[0]$ \\
Ag alloy & $43.3(2.8)[0]$ & $33.1(5.9)[0]$ & & $39.3(6.3)[0]$ & $40.5(4.0)[0]$ \\
SUS304 & $46.3(3.1)[0]$ & $38.2(2.7)[0]$ & & $45.6(5.7)[0]$ & $47.8(2.7)[0]$ \\
Co-Cr alloy & $39.3(2.6)[0]$ & $32.7(6.0)[0]$ & & $31.7(5.0)[0]$ & $40.8(4.3)[0]$ \\
Ni-Cr alloy & $42.6(3.6)[0]$ & $40.1(3.7)[0]$ & & $36.9(3.6)[0]$ & $43.0(2.3)[0]$ \\
Ti & $39.2(3.8)[0]$ & $43.6(3.8)[0]$ & & $38.6(7.6)[0]$ & $44.8(6.0)[0]$ \\
\hline
\end{tabular}

The values in brackets are the number of specimens fractured while thermal cycling, and the specimens are excluded from the calculation of the average and standard deviation.

Treatment agent remaining on the metal surface was washed off before the application of resin.

Tensile bond strength after 2,000 thermal cyclings in water at 4 and $60^{\circ} \mathrm{C}$.

( ): SD 


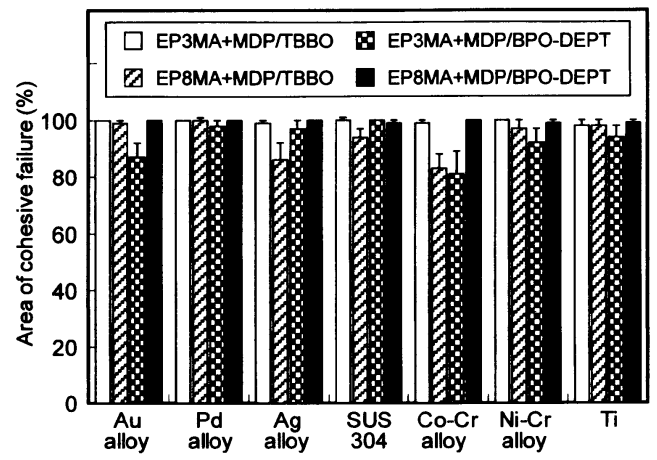

Fig. 2 The relative proportion of the area of cohesive failure after tensile tests for specimens treated with both a thiirane monomer and MDP in combination.

Column height and vertical line indicate mean and standard deviation, respectively.

alloy $(\mathrm{p}<0.05)$. Microscopic observation of debonded surfaces suggested that the failure mode of TBBO-initiated resin was nearly completely cohesive, whereas BPODEPT-initiated resin revealed primary cohesive and partial adhesive failures of less than 20\%, except for Pd alloy, Ag alloy and SUS304 which were nearly completely cohesive.

Combined surface treatment with EP8MA and MDP greatly enhanced the bond strengths to non-precious and precious metal alloys in the same manner as above. In contrast to the above case, BPO-DEPT resin demonstrated values of mean bond strength higher than TBBO resin, with the exception of $\mathrm{Au}$ alloy which exhibited insignificant differences between the two initiators $(p>0.05)$. The difference in bond strength between the two initiators was significant for Pd alloy, Ag alloy, SUS304, and $\mathrm{Co}-\mathrm{Cr}$ alloy $(\mathrm{p}<0.05)$. The fractured surfaces of BPO-DEPT-initiated resin after the tensile test demonstrated nearly completely cohesive failures of the resin layer in all cases. On the other hand, failures of TBBO-initiated resin were classified into the following two modes: Nearly complete cohesive failures for $\mathrm{Au}$ alloy, $\mathrm{Pd}$ alloy, $\mathrm{Ni}-\mathrm{Cr}$ alloy and $\mathrm{Ti}$; a combination of a cohesive failure and a partial adhesive failure of less than $20 \%$ for $\mathrm{Ag}$ alloy, SUS304 and Co-Cr alloy.

\section{DISCUSSION}

Adhesive bonding promoters for precious metals

Four kinds of epithioalkyl methacrylates have been prepared as adhesive bonding promoters for precious metals ${ }^{15,19)}$. The strong affinity to precious metals and excellent shelf life of thiirane monomers were considered to be derived from chemical 
activities such as a precursor of a thiol compound ${ }^{16)}$. The monomers differ in the length of their alkylene side chain, which affects water durability and the self-assembled structure of adsorbed molecules. It was reported that the contact angle of water on the methyl surface of a series of dialkyl sulfides with the $\mathrm{CH}_{3}-\mathrm{CH}_{2} \gamma_{\mathrm{n}} \mathrm{S}+\mathrm{CH}_{2} \gamma_{\mathrm{n}} \mathrm{CH}_{3}$ structure, which was adsorbed on gold, increased with increasing length of the alkylene group up to $n=6$, and that it was held constant thereaf$\operatorname{ter}^{21)}$. This phenomenon suggests that the dialkyl sulfides form highly ordered, closepacked monolayers at a value of $\mathrm{n}$ greater than 6 . Therefore, tight self-assembled structures on adherend substrates are expected for thiirane monomers with an alkylene chain longer than 6. Thus, EP8MA with an octamethylene chain was employed as an adhesive monomer for precious metals, and EP3MA with a propylene chain was used for comparison.

To obtain maximum performances of surface treatment agents, the conventional standard method employed agents with high concentration in ethanol and relatively long treatment durations, that is, 1 mol\% of solution was applied for one day ${ }^{15}$. The solvent and the concentration of the surface treatment agent were modified from the conventional one, since solutions containing both $1 \mathrm{~mol} \%$ of thiirane monomer and $1 \mathrm{~mol} \%$ of MDP were too concentrated to be used as a primer and MDP was more stable in acetone than in ethanol. Adherend metal surfaces were polished to a mirror-like finish to eliminate mechanical factors affecting bond strengths, since this investigation evaluated the chemical effect of adhesive bonding promoters. Therefore, the mechanical additive effects as well as the effect caused by the increased interface area would be expected if mechanical retentive treatment such as grit blasting was applied to adherend surfaces before the application of the surface treatment agent.

As shown in Table 3, the tensile bond strength of MMA-PMMA resins to precious metal alloys was markedly improved by the treatment with either EP3MA or EP8MA, although the lowest bond strength was recorded for Ag alloy among the precious metal alloys without exception. The difference in bond strength between Ag alloy and the other precious metal alloys was highly significant for EP3MA $(p<0.001)$, and significant for EP8MA $(p<0.05)$ with the exception of TBBO-initiated resin. The failure modes after tensile tests of thiirane-treated $\mathrm{Au}$ alloy or $\mathrm{Pd}$ alloy were nearly completely cohesive except for TBBO-initiated resin with EP8MA treatment, while Ag alloy demonstrated a combination of a cohesive failure and a partial adhesive failure at the peripheral region of the adhesion interface. It may possibly be the cause of this phenomenon that the affinity of the thiirane monomers to pure silver was slightly inferior to that of pure gold or palladium, as was the case of thiophenol ${ }^{7)}$.

Taking a wide view of these results, $0.1 \mathrm{~mol} \%$ of thiirane monomer acetone solution showed a similar tendency in bonding behavior to the case of $1 \mathrm{~mol} \%$ of thiirane monomer ethanol solution ${ }^{15,19)}$. The kind and concentration of solvent used for surface treatment agents appeared not to significantly affect the bond strength, since the solvent nearly fully evaporated while thiirane-applied surfaces were left to stand. 
Surface treatment using both an adhesive bonding promoter for non-precious metals and one for precious metals in combination

It was suggested that the improvement of the bond strength to non-precious metal alloys by the use of MDP is based on chemical bonding with the metal oxide formed on these alloys ${ }^{22)}$. The effectiveness of MDP treatment for precious metal alloys, as shown in Table 2, is not surprising in view of the fact that the precious metal alloys used here contain several kinds of base metal ingredients. However, the bond strength to precious metal alloys was considered to be below the satisfactory level compared with that of typical adhesive bonding promoter for precious metals. Thus, the multiple effects obtained by the combined use of an adhesive bonding promoter for precious metals and one for non-precious metals appeared to be an easy solution to a surface treatment agent for all kinds of dental metals. Based on similar reasoning, surface treatment agents containing both 6-[N-(4-vinylbenzyl)propylamino]1,3,5-triazine-2,4-dithione (VBATDT) and MDP were applied to dental alloys, and although the bond strength to non-precious metal alloys attained values above 40 $\mathrm{MPa}$ at the most suitable ratio of the two adhesive monomers ${ }^{23)}$, the bond strengths to precious metal alloys hardly exceeded $30 \mathrm{MPa}^{23)}$. On the other hand, the usage of both a thiirane monomer and 4-methacryloyloxyethoxycarbonylphthalic anhydride (4META) did not improve the bond strength to non-precious metal alloys up to 30 $\mathrm{MPa}^{24)}$. Therefore, it appeared to be most advantageous to combine a thiirane monomer and MDP.

As seen in Table 4, the tensile bond strengths to seven kinds of metal adherends were improved satisfactorily by the combined use of either EP3MA-MDP or EP8MAMDP without any spontaneous failures while thermal cycling. The mean bond strength exceeded $30 \mathrm{MPa}$ in all cases, and the usage of both EP8MA and MDP together with BPO-DEPT-initiated resin was particularly effective, resulting in bond strengths above $40 \mathrm{MPa}$. Such high performances appeared to be exhibited for the first time under the same experimental conditions. The initiator system of resin influenced the effectiveness of the combined use of two adhesive monomers, and the effects were dependent on the molecular structure of thiirane monomer. The mean bond strength obtained by the combined use of EP3MA and MDP was generally higher for MMA-PMMA/TBBO resin than for MMA-PMMA/BPO-DEPT resin, and the values obtained by the combined use of EP8MA and MDP were conversely higher for MMA-PMMA/BPO-DEPT resin.

Since the failure mode after the tensile test of these specimens was almost cohesive within the resin near the adhesion interface, the bond strengths were substantially limited by the tensile strength of the resin itself. The mechanical properties of MMA-PMMA resin are greatly affected by the initiator used, and in general, BPODEPT-initiated resin demonstrates strengths higher than TBBO-initiated one ${ }^{25)}$. Consequently, BPO-DEPT-initiated resins give relatively higher bond strengths compared with TBBO-initiated resins unless the polymerization of resin proceeds without any interference of ingredients in the surface treatment agent ${ }^{26)}$. Phosphoric acid monomer has a highly inhibitory action against the initiation reaction by BPO-amine 
initiator system, resulting in the development of BPO-amine-sulfinic acid ternary initiator system ${ }^{27)}$, while TBBO is not affected by the existence of phosphoric acid monomer. Therefore, BPO-DEPT initiator has an adverse effect on the polymerization in the presence of MDP, which leads to incompletely cured resin, and has the advantage of a stronger tensile strength of cured resin compared with TBBO initiator in the absence of MDP. The two inconsistent effects are considered to partially compensate each other according to the adsorbed molecular structure of MDP on the treated surface.

The following clarification offers a reasonable explanation for the above results. In the case of the combined use of EP8MA and MDP, since both monomers formed densely packed layers on the adherend surface due to the strong lateral hydrophobic interaction of long alkylene chains in EP8MA or MDP, phosphoric acid groups of MDP were tightly covered by self-assembled alkylene moieties, and the adverse effect of MDP was suppressed, resulting in the higher bond strength for BPO-DEPTinitiated resin. On the other hand, EP3MA and MDP formed less ordered monomer layers because of the lack of long alkylene chain in EP3MA, and, consequently, phosphoric acid groups of MDP were able to interact partially with BPO-DEPT initiator to produce weak polymer layers near the adhesion interfaces which decreased relatively the bond strength of BPO-DEPT-initiated resin compared with TBBO-initiated resin.

Whether this interpretation is generally acceptable cannot be known until further studies concerning the concentration dependence of thiirane monomer and MDP used in combination are performed, since the detailed adsorbed structures of coexisting thiirane monomer and MDP are not yet clarified.

\section{Multiple effects of the combined use of both a thiirane monomer and MDP}

The multiple effects of the combined treatment with EP3MA and MDP were clearly demonstrated for TBBO-initiated resin because the value of mean bond strengths of EP3MA-MDP treatment was never lower than that of EP3MA or MDP treatment with only one exception, the Au alloy, for which the value of EP3MA-MDP treatment was only 0.1 MPa smaller than that of EP3MA. On the other hand, the bond strength of BPO-DEPT-initiated resin to Au alloy treated with EP3MA-MDP was significantly decreased compared with that using EP3MA $(p<0.01)$. This may be caused by the adverse effect of MDP, that is, the above-mentioned inhibitory effect. There was no significant difference in bond strength to non-precious metal and alloys between EP3MA-MDP and MDP treatments regardless of the initiators except for TBBO-initiated resin to Co-Cr alloy (for TBBO-initiated resin to $\mathrm{Co}-\mathrm{Cr}$ alloy; $\mathrm{p}<0.01$, for others; $p>0.05$ ). Therefore, the EP3MA component of the EP3MA-MDP treatment agent appeared to hardly affect the bond strength to non-precious metal and alloys and to behave similar to solvents.

The value of the mean bond strengths of BPO-DEPT-initiated resin to metal adherends treated with EP8MA-MDP agent was always larger than that with onecomponent agents. The combined use of EP8MA and MDP significantly improved the 
bond strength to even non-precious metal alloys such as SUS304 and Co-Cr alloys compared with the usage of MDP when MMA-PMMA/BPO-DEPT resin was used $(p<0.05)$. This unexpected finding may be due to the lateral hydrophobic interactions of long alkylene chains between EP8MA and MDP resistant against the attack of BPO-DEPT initiator, since the treatment with EP8MA alone was quite ineffective in promoting the bond strength to these non-precious metal alloys.

On the other hand, the effect of EP8MA-MDP combined treatment on the bond strength of TBBO-initiated resin did not show a decisive tendency in contrast to BPO-DEPT-initiated resin and the combined usage was rather disadvantageous in bond strengths to Ag alloy, SUS304, Ni-Cr alloy, compared with the treatment with MDP alone, although only a difference in bond strength to SUS304 between MDP and EP8MA-MDP treatments was significant $(p<0.01)$. The finding that the bond strength to these non-precious metal alloys treated with MDP alone was higher for TBBO-initiated resin than for BPO-DEPT-initiated resin may be one apparent reason for the above disadvantageous reduction in bond strength, and detailed information on the geometry of adsorbed monomers is needed to clarify the difference in adhesion behavior between these initiators.

Another method for evaluating bonding durability against water was reported ${ }^{4,28)}$. The relative proportion of the cohesive and adhesive failures for the total cross-sectional area of the fractured specimen after the tensile test is proportionally correlated to the magnitude of the tensile bond strength as long as there is a distinct borderline between cohesive and adhesive failures ${ }^{12)}$. This relative proportion serves as a convenient evaluation method for bonding durability against water when adhesive resins with different cohesive strengths are compared, since the proportion does not depend on cohesive strength of the resin itself but depends on interfacial durability against water.

The area of adhesive failure spread out to a central area, as water durability decreased. The phenomenon suggested that the bond between resin and metal was disrupted by water molecules from the periphery toward the center at the interface. The percentage of the cohesive failure in the cases of combined treatments was almost $100 \%$ for all in TBBO-initiated resin with EP3MA-MDP treatment and for all in BPO-DEPT-initiated resin with EP8MA-MDP treatment. For the other specimens with the combined treatments, the values changed within the range of $80 \%$ and $100 \%$, approximately in proportion to the obtained bond strength. In general, the percentage of cohesive failure reached almost $100 \%$ when the bond strength was greater than about $39 \mathrm{MPa}$, and the value decreased to reach the minimum value of about $80 \%$ as the bond strength decreased to about $30 \sim 32 \mathrm{MPa}$ in the cases of the combined treatments. However, no distinct difference in the relation of the percentage of cohesive failure and bond strength was observed between TBBO-initiated resin and BPO-DEPT-initiated resin due to the lack of sufficient accuracy of the area measured. The overall effectiveness of the surface treatments based on microscopic failure mode analysis was improved in the following order: MDP /BPO-DEPT $<$ $\mathrm{MDP} / \mathrm{TBBO}<\mathrm{EP} 3 \mathrm{MA}-\mathrm{MDP} / \mathrm{BPO}-\mathrm{DEPT} \approx \mathrm{EP} 8 \mathrm{MA}-\mathrm{MDP} / \mathrm{TBBO}<\mathrm{EP} 3 \mathrm{MA}-\mathrm{MDP} / \mathrm{TBBO} \approx$ 
EP8MA-MDP/BPO-DEPT.

The tensile test and failure mode analysis suggested that TBBO-initiated resin with EP3MA-MDP treatment and BPO-DEPT-initiated resin with EP8MA-MDP treatment were the most effective surface treatment system to promote the water durability of bonds between an adhesive resin and a dental metal. The combined use of EP8MA and MDP is expected to have very great utility in surface modification of dental metals, since EP3MA possesses a peculiar odor and is fully evaporated when the EP3MA treatment agent on the adherend surface was left to stand for one day, while EP8MA is an odorless non-volatile liquid. Taking into consideration the inhibitory action of MDP against the initiation reaction of BPO-amine initiator system, EP8MA-MDP treatment using BPO-amine-sulfinic acid ternary initiator system would be also necessary study.

\section{CONCLUSION}

Five surface treatment agents containing MDP, EP3MA, EP8MA, EP3MA-MDP, or EP8MA-MDP were prepared by dissolving monomers in acetone. The surfaces of seven kinds of dental metals were treated by the treatment agent and then metal specimens were butt-jointed. After 2,000 thermal cyclings in water, the tensile bond strengths were measured. The following conclusions can be drawn from the results of the tensile tests and the microscopic failure mode analysis.

(1) The surface treatment with 0.1 mol\% of MDP acetone solution markedly improved the bond strengths to non-precious metal alloys or $\mathrm{Ti}$ without any spontaneous failures while thermal cycling, and was shown to be effective to some extent for precious metal alloys.

(2) The tensile bond strength to precious metal alloys was significantly enhanced by EP3MA or EP8MA treatment but no effect was demonstrated when non-precious metal alloys or $\mathrm{Ti}$ were employed as an adherend.

(3) The bond strength obtained by EP3MA-MDP treatment was generally higher for MMA-PMMA/TBBO resin than for MMA-PMMA/BPO-DEPT resin, and the value obtained by EP8MA-MDP treatment was conversely higher for MMA-PMMA/BPODEPT resin.

(4) The combined treatment of EP8MA and MDP by the use of BPO-DEPT-initiated resin was significantly effective compared with EP8MA or MDP treatment and the mean bond strength to dental metals exceeded $40 \mathrm{MPa}$ in all cases.

(5) The overall effectiveness of surface treatments based on microscopic failure mode analysis was improved in the following order: MDP / BPO-DEPT $<$ MDP / TBBO < EP3MA-MDP / BPO-DEPT $\approx$ EP8MA-MDP / TBBO <EP3MA-MDP / TBBO EP8MA-MDP/BPO-DEPT.

\section{REFERENCES}

1) Tanaka, T., Atsuta, M., Nakabayashi, N. and Masuhara, E.: Surface treatment of gold alloys for adhesion, $J$ Prosthet Dent 60 : 271-279, 1988. 
2) Yamashita, A., Kondo, Y. and Fujita, M.: Adhesive strength of adhesive resin PANAVIA EX to dental alloys, $J$ J Prosthodont Soc $28: 1023-1033$, 1984. (in Japanese)

3) Tiller, J., Gobel, R., Magnus, B., Musil, R. and Jena, R. B.: Werkstoffkundliche Grundlagen zum Silicoater-MD-Verfahren, Dental Labor 38 : 78-82, 1990.

4) Ohno, H., Araki, Y. and Endo, K.: A new method for promoting adhesion between precious metal alloys and dental adhesives, J Dent Res 71:1326-1331, 1992.

5) Colin, D. B., Troughton, E. B., Tao, Y-T., Evall, J., Whitesides, G. M. and Nuzzo, R. G.: Formation of monolayer films by the spontaneous assembly of organic thiols from solution onto gold, J Am Chem Soc 111 : 321-335, 1989.

6) Toriyama, H., Wakabayashi, H., Kato, T., Imai, M., Kondo, Y., Yamashita, A., Kawashima, M., Tsugaru, T. and Omura, I.: Pre-treatment of dental alloys using adhesive primer for precious alloys, $J J$ Dent Mater $10: 739-747,1991$. (in Japanese)

7) Kojima, K.: Studies on adhesion of functional monomers with $\mathrm{SH}$ group to tooth substrates and dental alloys, $J$ J Dent Mater $5: 92-105$, 1986. (in Japanese)

8) Kojima, K. and Kadoma, Y.: Surface treatment of metal with disulfide monomer - Eff ect of time of treatment and tensile bond strength-, $J J$ Dent Mater $12: 122-123$, 1993. (in Japanese)

9) Kojima, K., Kadoma, Y. and Imai, I.: Adhesion to precious metal using new type functional monomer containing sulfur, $J J$ Dent Mater $6: 112-113$, 1987. (in Japanese)

10) Kadoma, Y.: Application of 5-(4-vinylbenzyl)-2-thiobarbituric acid to dental materials, $J$ $J$ Dent Mater 11 : 891-898, 1992. (in Japanese)

11) Kojima, K., Kadoma, Y. and Imai, I.: Adhesion to precious metals utilizing triazinedithione derivative monomer, $J J$ Dent Mater $6: 702-707$, 1987. (in Japanese)

12) Kadoma, Y. and Kojima, K.: Adhesive properties of MMA-PMMA/TBBO resin to dental alloys treated with sulfide-containing monomer, $J J$ Dent Mater 11:940-946, 1992. (in Japanese)

13) Kojima, K. and Kadoma, Y.: Surface treatment with acrylate monomers derived from 1,2-dithiolane - Its effect on adhesion of resin to dental precious metals-, $J J$ Dent Mater $16: 126,1997$. (in Japanese)

14) Matsumura, H., Atsuta, M. and Nakamura, M.: Bonding of a silver-palladium-coppergold alloy with a thiouracil derivative primer, $A D 16: 82-87,1998$. (in Japanese)

15) Kadoma, Y.: Surface treatment of precious metal alloys with thiirane monomer and the effect on the bond strength of resin, $J J$ Dent Mater $15: 446-453$, 1996. (in Japanese)

16) Suzuki, M., Miyazaki, T., Hisamitsu, H., Kadoma, Y. and Morioka, Y.: Study on chemical reaction of methylthiirane on gold colloid by surface-enhanced Raman scattering, Langmuir 15 : 7409-7410, 1999.

17) Kadoma, Y.: Adhesion to dental precious metal alloys using MMA-PMMA resin containing a thiirane monomer, $J J$ Dent Mater $17: 200-206$, 1998. (in Japanese)

18) Omura, I. and Yamauchi, J.: Correlation between molecular structure of adhesive monomer and adhesive properties, in Transactions of International Congress on Dental Materials, 356(P40), 1989.

19) Kadoma, Y.: Surface treatment of dental precious metal alloys by thiirane monomers having a long alkylene chain, $J J$ Dent Mater $16: 114-121$, 1997. (in Japanese)

20) Nealon, F. H.: Acrylic restorations by the operative nonpressure procedure, $J$ Pros Dent $2: 513-527,1952$.

21) Troughton, E. B., Bain, C. D., Whitesides, G. M., Nuzzo, R. G., Allara, D. L. and Porter, M. D.: Monolayer films prepared by the spontaneous self-assembly of symmetrical and unsymmetrical dialkyl sulfides from solution onto gold substrates: Structure, properties, and reactivity of constituent functional groups, Langmuir $4: 365-385,1988$.

22) Matsumura, H., Taira, Y. and Atsuta, M.: Adhesive bonding of noble metal alloys with a triazine dithiol derivative primer and an adhesive resin, $J$ Oral Rehabil 26:877-882, 1999.

23) Kojima, K., Kadoma, Y. and Yamauchi, J.: Study on adhesive primer to dental metal 
used for composite type resin cement, $J J$ Dent Mater $16: 316-321,1997$. (in Japanese)

24) Kadoma, Y.: Adhesion of resin to dental alloys treated by both thiirane monomer and 4-META, $J J$ Dent Mater $19: 301-309,2000$. (in Japanese)

25) Chen, S-Y., Kojima, K., Kadoma, Y. and Masuhara, E.: The effects of the conditions of polymerization on the various properties of MMA-PMMA resin, $J J$ Dent Mater 4:356362, 1985. (in Japanese)

26) Kadoma, Y.: Surface treatment of metals using thiobarbituric acid monomers - The durability of adhesion of MMA-PMMA/BPO-amine resin-, $J J$ Dent Mater 14:393-398, 1995. (in Japanese)

27) Kawashima, M. and Yamauchi, J., DE $133: 35-38,2000$. (in Japanese)

28) Antoniadou, M., Kern, M. and Strub, J. R.: Effect of a new metal primer on the bond strength between a resin cement and two high-noble alloys, J Prosthet Dent $84: 554-560$, 2000 . 